\title{
Development of a Detailed Surface Chemistry Framework in DSMC
}

\author{
K. Swaminathan-Gopalan, ${ }^{\text {a* A. Borner, }}{ }^{\mathrm{b}}$ K. A. Stephani ${ }^{\mathrm{a}}$ \\ ${ }^{a}$ Department of Mechanical Science and Engineering, University of Illinois at Urbana-Champaign, \\ Urbana, IL 61801 USA \\ ${ }^{\mathrm{b}}$ Science and Technology Corporation at NASA Ames Research Center, Moffett Field, CA 94035 USA \\ *kswmnth2@illinois.edu
}

Many of the current direct simulation Monte Carlo (DSMC) codes still employ only simple surface catalysis models. These include only basic mechanisms such as dissociation, recombination, and exchange reactions, without any provision for adsorption and finite rate kinetics. Incorporating finite rate chemistry at the surface is increasingly becoming a necessity for various applications such as high speed re-entry flows over thermal protection systems (TPS), micro-electro-mechanical systems (MEMS), surface catalysis, etc. In the recent years, relatively few works have examined finite-rate surface reaction modeling using the DSMC method., ${ }^{2,3}$

In this work, a generalized finite-rate surface chemistry framework incorporating a comprehensive list of reaction mechanisms is developed and implemented into the DSMC solver SPARTA. ${ }^{4}$ The various mechanisms include adsorption, desorption, Langmuir-Hinshelwood (LH), Eley-Rideal (ER), Collision Induced (CI), condensation, sublimation, etc. The approach is to stochastically model the various competing reactions occurring on a set of active sites. Both gas-surface (e.g., ER, CI) and pure-surface (e.g., LH, desorption) reaction mechanisms are incorporated. The reaction mechanisms could also be catalytic or surface altering based on the participation of the bulk-phase species (e.g., bulk carbon atoms). Marschall and MacLean ${ }^{5}$ developed a general formulation in which multiple phases and surface sites are used and we adopt a similar convention in the current work. Microscopic parameters of reaction probabilities (for gas-surface reactions) and frequencies (for pure-surface reactions) that are require for DSMC are computed from the surface properties and macroscopic parameters such as rate constants, sticking coefficients, etc. The energy and angular distributions of the products are decided based on the reaction type and input parameters. Thus, the user has the capability to model various surface reactions via user-specified reaction rate constants, surface properties and parameters.
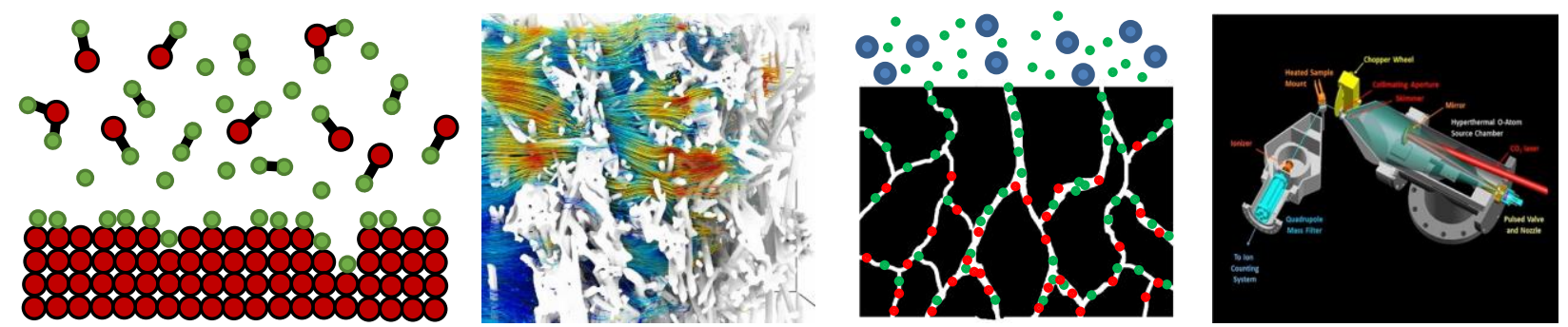

Figure 1. Some common applications of surface chemistry using DSMC (a) General schematic of adsorption and reactive surface chemistry. (b) Velocity streamlines from the flow inside carbon fiber preform material used as TPS. (c) Microporous catalyst used for species selectivity. (d) Schematic of molecular beam experimental setup. ${ }^{6}$

[1] G. A. Bird, Molecular Gas Dynamics and the Direct Simulation of Gas Flows, Oxford University Press, Oxford, UK (1994).

[2] I. Choquet, "A new approach to model and simulate numerically surface chemistry in rarefied flows," Physics of Fluids, vol. 11, no. 6, p. 1650, Jun 1999.

[3] A. N. Molchanova et al., "A detailed DSMC surface chemistry model," AIP Conference Proceedings, vol. 1628, no. 131, pp. 131-138, 2014.

[4] M. A. Gallis, et al., "Direct simulation monte carlo: The quest for speed," AIP Conference Proceedings, vol. 1628, no. 1, pp. 27-36, 2014.

[5] J. Marschall and M. MacLean, "Finite-rate surface chemistry model, I: Formulation and reaction system examples," in 42nd AIAA Thermophysics Conference, 2011, p. 3783.

[6] V. J. Murray, et al., "Inelastic and Reactive Scattering Dynamics of Hyperthermal O and $\mathrm{O}_{2}$ on Hot Vitreous Carbon Surfaces," Journal of Physical Chemistry C, vol. 119, no. 26, pp. 14780-14796, 2015.

This work was performed under the Entry System Modeling Project (M. J. Wright Project Manager) at the NASA Game Changing Development (GCD) Program and supported by NASA Grant NNX15AU92F. 